\title{
The excitatory effect of antioxidant and immune polysaccharide Fig
}

\author{
Ming Bai, Weiyun Xin, Mingsan Miao ${ }^{a}$, Xiaoyan Fang and Can Wang \\ Department of Pharmacology, Henan University of Traditional Chinese Medicine, Zhengzhou 450008, China
}

\begin{abstract}
Objective: To investigate the Ficus carica polysaccharide (FCPS) antioxidant and immune excited. Method: Made metabolism in aging model mice of D-galactose, observe the effect of FCPS on the aging model in mice red blood cell Superoxide dismutase (SOD), Catalase (CAT) and Glutathione peroxidase (GSH-Px) activity and lipid peroxidation in the serum, liver homogenate, brain homogenate (LPO) level. Cyclophosphamide (CY) caused by mice immunosuppression model, observation of FCPS on peritoneal macrophage phagocytic percentage and phagocytic index, the formation of hemolysin and hemolysis, effects of lymphocyte transformation. Two experiments were mushrooms polysaccharide as positive drug. Results: FCPS could significantly improve the aging model mice of SOD, CAT, GSH-Px levels $(\mathrm{P}<0.05)$, and the decrease of plasma, liver homogenate and brain homogenate LPO levels $(\mathrm{P}<0.01)$; enhance the peritoneal macrophage phagocytic percentage and phagocytic index $(\mathrm{P}<0.01)$, promote the formation of hemolysin and hemolytic plaque and the transformation of lymphocyte. Conclusion: FCPS could improve the immune function of immunosuppressive mice, enhance the antioxidant capacity of aging model mice.
\end{abstract}

\section{Introduction}

Fig (FC) is the Ficus hispida plants, sweet natured, with tonifying spleen and stomach, lungs and cough, swelling detoxification of reactive power, and contains organic acids, carbohydrates, vitamin rich, for the homology of medicine and food product [1], because of its anticancer effect and better nutritional value and caused widespread concern. Modern research shows that polysaccharide is the main active component of Fig water soluble part, studies have found that FCPS can significantly improve the immune function of immunosuppressive mice, promote the recovery of immune function [2]. Pharmacological studies have confirmed that the latex, fig juice fragrant ingredients, psoralen and bergamot lactone for its main anticancer active ingredients. In addition, the data show the carcinogenesis associated with oxidative effect, antitumor effect of drugs is also its antioxidant effects exist certain relationship [3]. Chinese medicine in the prevention and treatment of cancer in addition to direct inhibitory effect on tumor, excitatory effect on immune function is its role in preventing tumor in another important way. Existing research shows [4-7]: Fig leaves and fruits contain high concentrations of superoxide dismutase (SOD), flavonoids, vitamin C (Vc) and other substances, the fig plant has a good antioxidant, anti-aging effect. On the basis of all these discussions extract of FCPS as the research object, observe its antioxidation and immune exciting role, providing a basis for the further discussion for its anticancer function.

\section{Materials}

\subsection{Reagents drugs}

D-galactose, Shanghai reagent two factory production; cyclophosphamide, Shanghai twelve pharmaceutical factory production; thiobarbituric acid (TBA), Shanghai reagent factory production of hydrogen peroxide, two; Shanghai Yao $\mathrm{Pu}$ chemical factory production; SOD kit, Nanjing Jiancheng Biological Engineering Research Institute of production; plant hemagglutinin, clinical science and technology Shanghai Yi Hua company. Fig was purchased from Medicines Co in Henan Province, by the Institute of chemical analysis room isolation and extraction of polysaccharide of $\mathrm{FC}$ (polysaccharide content of about 95\%) identified by the Department identified as genuine; mushrooms polysaccharide tablets, Jiangsu province Jiangyin pharmaceutical factory production.

\subsection{Animal}

Kunming (KM) mice, weight 20-22 g, male and female; the British cavy, were provided by medical experimental animal center of Henan province. (animal license number, SCXK (Henan) 2010-0002 )

\section{Methods}

\subsection{Antioxidative effect of FCPS}

Took 50 mice, were randomly divided into 5 groups, 10 rats in each group, respectively, that is blank group,

a Corresponding author: miaomingsan@126.com 
model group, mushrooms polysaccharide group (MP), Ficus carica polysaccharide high, low dose group (FCPS-HD, FCPS-LD). Except the blank group, other groups daily subcutaneous injection of D-galactose, weigh 1 times every 3 days, according to the weight adjusted dose, administered continuously for 40 days [8], blank group were injected with the same volume of normal saline. From the eleventh day of FCPS-HD, FCPS-LD were $400 \mathrm{mg} / \mathrm{kg}, 200 \mathrm{mg} / \mathrm{kg}$ perfusion of FCPS solution, MP by $100 \mathrm{mg} / \mathrm{kg}$ gavage MP suspension, model group and blank group were given the same volume of saline, 1 times a day. In fortieth days morning injection of D-galactose, perfusion $2 \mathrm{H}$ mice after picking eye blood, heparin standby, preparation of homogenate from the brain and liver tissue system. Determination of the method was as follows: SOD activity assay: took anti clotting $50 \mu \mathrm{l}$, added $5 \mathrm{ml}$ saline filled with centrifugal tube, mixed, 3000 rotating centrifugal $5 \mathrm{~min}$, abandoned as supernatant, plus pre cooling double distilled water 0.2 $\mathrm{ml}$ hemolysis, and pre cooling $0.1 \mathrm{ml}$ of $95 \%$ ethanol, the oscillation for a moment, in the pre cold chloroform 0.1 $\mathrm{ml}$, oscillation and centrifuged, the supernatant was, according to kit measured SOD activity in red blood cells. The CAT activity determination: according to the literature method, the OD value of 0.53 at $230 \mathrm{~nm}$, could be used as substrate concentration catalase, took pre temperature substrate $25^{\circ} \mathrm{C} 2 \mathrm{ml}$, adding 1:100 hemolytic solution of $0.01 \mathrm{ml}$ under the condition of $25^{\circ} \mathrm{C}$, measured OD values in 230nm, measured 1 times per minute. GSH-Px activity assay: took the blood of the rats $20 \mathrm{~L}$, with double distilled water and diluted to $1.0 \mathrm{ml}$, according to the literature method in $422 \mathrm{~nm}$ measurement of each tube OD, in order to eliminate the influence of non enzyme system of GSH, another non enzymatic reaction control tube, the activity of GSH-Px was calculated by the method of literature. The determination result was shown in Table 1.

Serum LPO level in the top layer by Buege and other TBA chemical colorimetric method, the content of LPO were measured by the method of literature within the organization first homogenate, then measured the LPO levels in the organization, recording the absorbance (see from Table 2).

Table 1. Effects of FCPS on the aging model mice blood SOD, CAT and GSH-Px activity ( $\bar{x} \pm s)$.

\begin{tabular}{|c|c|c|c|c|c|}
\hline Groups & $n$ & $\begin{array}{c}\text { Dose } \\
(\mathrm{mg} / \mathrm{kg})\end{array}$ & $\begin{array}{c}\text { SOD } \\
(\mathrm{NU} / \mathrm{ml})\end{array}$ & $\begin{array}{c}\text { CAT } \\
\left(\mathrm{S}^{-1} \times 10^{-4}\right)\end{array}$ & $\begin{array}{c}\text { GSH-Px } \\
(\mathrm{U} / \mathrm{ml} / \mathrm{min})\end{array}$ \\
\hline Blank group & 10 & -- & $1335 \pm 377^{1)}$ & $6.354 \pm 1.353^{2}$ & $17.14 \pm 4.52^{2)}$ \\
\hline Model group & 10 & -- & $892 \pm 350$ & $3.878 \pm 1.037$ & $8.56 \pm 2.20$ \\
\hline MP & 10 & 100 & $1709 \pm 553^{2)}$ & $5.239 \pm 1.323^{1)}$ & $36.00 \pm 2.50^{2}$ \\
\hline FCPS-HD & 10 & 400 & $1609 \pm 649^{2}$ & $6.982 \pm 1.384^{2)}$ & $17.53 \pm 3.49^{2}$ \\
\hline FCPS-LD & 10 & 200 & $1537 \pm 500^{2)}$ & $6.036 \pm 1.493^{2)}$ & $23.30 \pm 3.22^{2)}$ \\
\hline
\end{tabular}

Note: compared with the model group, 1) $P<0.05$; 2) $P<0.01$.

Table 2. Effects of FCPS on the level of LPO in serum and brain/liver homogenate of aging model mice ( $\bar{x} \pm s$ ).

\begin{tabular}{cccccc}
\hline Groups & $n$ & $\begin{array}{c}\text { Dose } \\
(\mathrm{mg} / \mathrm{kg})\end{array}$ & Plasma & Brain homogenate & Liver homogenate \\
\hline Blank group & 10 & -- & $0.064 \pm 0.012^{1^{1}}$ & $0.048 \pm 0.011^{1^{1}}$ & $0.054 \pm 0.010^{1^{1}}$ \\
Model group & 10 & -- & $0.129 \pm 0.029$ & $0.104 \pm 0.017$ & $0.160 \pm 0.028$ \\
MP & 10 & 100 & $0.115 \pm 0.017$ & $0.075 \pm 0.014^{1)}$ & $0.095 \pm 0.012^{1^{1}}$ \\
FCPS-HD & 10 & 400 & $0.091 \pm 0.019^{1)}$ & $0.054 \pm 0.008^{1)}$ & $0.075 \pm 0.011^{1^{1}}$ \\
FCPS-LD & 10 & 200 & $0.087 \pm 0.021^{1)}$ & $0.061 \pm 0.009^{1)}$ & $0.087 \pm 0.019^{1^{1}}$ \\
\hline
\end{tabular}

Note: compared with the model group, 1) $P<0.01$.

Table 3. Effect of FCPS on CY induced immunosuppressed mice celiac macrophage phagocytic and lymphocyte transformation $(\bar{x} \pm s)$.

\begin{tabular}{|c|c|c|c|c|c|}
\hline Groups & $n$ & $\begin{array}{c}\text { Dose } \\
(\mathrm{mg} / \mathrm{kg})\end{array}$ & $\begin{array}{c}\text { Phagocytic percentage } \\
(\%)\end{array}$ & phagocytic index & $\begin{array}{c}\text { lymphocyte } \\
\text { transformation } \\
(\%)\end{array}$ \\
\hline Blank group & 10 & -- & $42.3 \pm 2.0^{1}$ & $0.58 \pm 0.06^{1}$ & $40.6 \pm 3.8^{1)}$ \\
\hline Model group & 10 & -- & $28.1 \pm 3.5$ & $0.40 \pm 0.04$ & $30.1 \pm 6.1$ \\
\hline MP & 10 & 100 & $35.5 \pm 3.5^{1)}$ & $0.49 \pm 0.07^{1)}$ & $49.0 \pm 5.6^{1)}$ \\
\hline FCPS-HD & 10 & 400 & $42.3 \pm 4.6^{1)}$ & $0.72 \pm 0.14^{1}$ & $54.3 \pm 6.8^{1)}$ \\
\hline FCPS-LD & 10 & 200 & $37.2 \pm 4.7^{1}$ & $0.56 \pm 0.10^{1)}$ & $49.4 \pm 6.8^{1)}$ \\
\hline
\end{tabular}

Note: compared with the model group 1) $P<0.01$ 
Table 4. Effect of FCPS on CY induced immunosuppressed mice hemolysin and hemolytic plaque formation $(\bar{x} \pm S)$.

\begin{tabular}{ccccc}
\hline Groups & $n$ & Dose $(\mathrm{mg} / \mathrm{kg})$ & $\begin{array}{c}\text { Hemolysin formation } \\
(\text { OD })\end{array}$ & $\begin{array}{c}\text { Hemolytic plaque formation } \\
(\text { OD) }\end{array}$ \\
\hline Blank group & 10 & -- & $0.098 \pm 0.014^{2}$ & $0.140 \pm 0.025^{1}$ \\
Model group & 10 & -- & $0.043 \pm 0.008$ & $0.101 \pm 0.011$ \\
MP & 10 & 100 & $0.054 \pm 0.012^{1)}$ & $0.147 \pm 0.020^{2}$ \\
FCPS-HD & 10 & 400 & $0.066 \pm 0.018^{2}$ & $0.130 \pm 0.023^{1}$ \\
FCPS-LD & 10 & 200 & $0.067 \pm 0.019^{2}$ & $0.144 \pm 0.028^{\prime}$
\end{tabular}

Note: compared with the model group, 1) $P<0.05$; 2) $P<0.01$

\subsection{Excited immune function of FCPS}

Grouping with the same as anti-oxidation experiment. The MP and model group, FCPS-HD, FCPS-LD were first, 2, 3 days according to the $80 \mathrm{mg} / \mathrm{kg}$ ip new preparation of CY physiological saline solution [9]. At the same time give mice fed FCPS-HD, FCPS-LD were $400 \mathrm{mg} / \mathrm{kg}, 200 \mathrm{mg} / \mathrm{kg}$ made the FCPS solution, MP according to the $100 \mathrm{mg} / \mathrm{kg}$ is made of the suspension, model group and blank group were given the same volume of saline, 1 times a day, for 8 consecutive days, on the eighth day observation each index.

\subsubsection{Effect of CY induced immunosuppressed mouse peritoneal macrophage phagocytosis function and the ymphocyte transformation}

On the eighth day morning, each experimental rat ip5\% chicken red blood cell(CRBC)physiological saline suspension, to CRBC physiological saline suspension after intragastric administration of $6 \mathrm{~h}$, the mice were sacrificed at $2 \mathrm{~h}$ after abdominal injection, disinfection, Han's solution $2.5 \mathrm{ml}$, gentle mice abdomen, peritoneal fluid is arranged in the slide, slide the bottom bunk with wet gauze, $37^{\circ} \mathrm{C}$ incubation $30 \mathrm{~min}$, normal saline flush to the floating cells, Wright's staining, washing drying, microscope and calculating the phagocytic percentage and phagocytic index [10] (see Table 3). Administration of the previous 3 days, each day mice were filled with PHA $10 \mathrm{mg} / \mathrm{kg}$, on the eighth day after administration of 2H mice tails blood, push plate, Wright's staining, oil microscope and calculating the percentage of lymphocyte transformation[10] (see Table 3).

\subsubsection{Effect of CY induced immunosuppressed mouse hemolysin and hemolytic plaque formation}

On the first day of administration, the CRBC physiological saline each mice abdominal cavity filling $5 \%$ suspension $0.2 \mathrm{ml}$ immunization. The mice were sacrificed after peritoneal fluid, took $1 \mathrm{ml}$ with physiological saline 1:100 after dilution, and 5\% CRBC mixed liquid of $0.5 \mathrm{ml}$, the $10 \%$ complement (guinea pig serum) $0.5 \mathrm{ml}$, mixed, $37^{\circ} \mathrm{C} 30 \mathrm{~min}$ of incubation, ice water to stop the reaction, the supernatant was in 72-1 spectrophotometer colorimetric $540 \mathrm{~nm}$, with a blank without complement the tube as the control, to determine the formation of hemolysin [10]. (results are shown in Table 4) the CRBC immunity in mice after the last administration of $2 \mathrm{H}$ spleen, 2 mice spleen together, with homogenate machine, regulating the spleen cell concentration of $5 \times 10^{6} / \mathrm{ml}$. Spleen cell suspension $0.5 \mathrm{ml}$, plus $0.2 \%$ CRBC suspension and guinea pig serum 1:10, $0.5 \mathrm{ml}$ respectively, mixed, $37^{\circ} \mathrm{C}$ incubation $1 \mathrm{H}$, centrifugation, supernatant from 72-1 spectrophotometer colorimetric $413 \mathrm{~nm}$, with a blank without complement tube zero, for the determination of hemolytic plaque formation [10]. (see Table 4)

\subsection{Statistical analysis}

The SPSS 13.0 statistical software for Windows was used for data analysis. The measurement results were expressed as mean \pm standard deviation $(\bar{x} \pm \mathrm{s})$. Comparison between groups was performed by the Ridit analysis.

\section{Results}

\subsection{Antioxidative effect of FCPS}

\subsubsection{Influence of mice blood SOD, CAT, GSH-Px}

It was seen from table 1 , in our current experimental conditions, compared with the blank group, the mice blood SOD, CAT and GSH-Px activity of model group,there was a statistically significant difference $(P<0.05)$, FCPS-HD, FCPS-LD could significantly improve the aging model mice blood SOD, CAT and GSH-Px activity, there was a statistically significant difference compared with the model group $(P<0.05)$.

\subsubsection{Influence on the level of LPO in serum and brain/liver homogenate of aging model mice}

As can be seen from table 2, plasma, brain homogenate in model group, liver homogenate LPO levels were significantly higher, there were significant differences compared with the blank group. Compared with model group, FCPS-HD, FCPS-LD could significantly reduce the plasma, liver, brain homogenate LPO levels.

\subsection{Excited immune function of FCPS}




\subsubsection{Inhibition of mice peritoneal macrophage phagocytosis function and the effect of the immune lymphocyte transformation induced by $\mathrm{CY}$}

It could be seen from Table 3, compared with the blank group, the phagocytic percentage and phagocytic index, lymphocyte transformation rate of model group had significant statistical significance $(P<0.01)$. Compared with the model group, FCPS-HD, FCPS-LD and MP could significantly improve the immune suppression mice peritoneal macrophage phagocytosis and phagocytic percentage scores, and promote the proliferation of lymphocytes $(P<0.01)$.

\subsubsection{Effect of FCPS on CY induced immunosuppressed mice hemolysin and hemolytic plaque formation}

It could be seen from Table 4, in the model group hemolysin and hemolytic plaque forming OD values were significantly lower than those in the blank group. Compared with the model group, the significant of FCPS-HD, FCPS-LD could promote the formation of hemolysin $(P<0.01)$, FCPS-HD could obviously promote the formation of plaque $(P<0.05)$.

\section{Discussion}

The metabolic theory of aging thik that metabolism disorders, aging is the result of. With the increasingly polluted environment, accelerate the pace of life, increasing pressure, people memory gradually decline, the increase of population aging. Fig is the homology of medicine and food products, for antitumor curative effect and no toxic side effect on the clinical side effects, but also can improve the radiotherapy and chemotherapy. According to reports, active oxygen and lipid peroxide (LPO) in the body, can cause oxidative DNA damage, thereby causing mutation and cancer, LPO also may be modified LDL approach to accelerate the formation and development of atherosclerosis. Therefore, the timely removal of reactive oxygen species in the body and reduce the LPO, can delay aging, prevent many diseases.

D-galactose is a physiological and nutritional ingredients, can be converted to glucose is involved in the metabolism of excess supply, but can lead to metabolic disorders. The study found that, for continuous high dose animal injection of D- galactose, causes and biochemical changes similar to natural aging, immune dysfunction, abnormal gene expression and regulation, cell growth and reproduction capacity decreased, cell degeneration and other cognitive performance of [11]. Previous study showed that, reduce the D-galactose induced SOD, GSH-Px activity, thereby causing the decline of brain function [12]. CY is a commonly used alkylating agent, has an inhibitory effect on the immune function. Therefore, this experiment used animal model of low immune function of CY. At present, the domestic and foreign research on physiological activity of FCPS mainly focused on anti tumor, analgesic, antibacterial and other aspects, the Research Report on the mechanism of antioxidant ability and other aspects also does not have the system [13]. It has been reported that the Fig water extracts have excited immunity and antitumor effect of polysaccharide of FC, but few studies have investigated.

In this study, experiments showed that the phagocytic index of FCPS-HD, FCPS-LD could significantly increase the CY induced immunosuppressed mice peritoneal macrophages and phagocytic percentage, significantly promote the formation of hemolysin and lymphocyte transformation, FCPS-HD could significantly promote the formation of plaque. It suggests that the immune of FCPS on low excitatory effect of good. One of the mechanism of FCPS immune excitatory effects may also be fig of prevention and treatment of cancer. In addition, FCPS content in the Fig is higher, is the main component of immune effect of Fig excited.SOD is a widely distributed in living organisms, and cellular oxygen metabolism closely related enzyme, is the main body of the enzyme superoxide anion radical scavenging activity, SOD reflects the antioxidant ability[11]. SOD, CAT, GSH-Px is an important antioxidant enzymes activity, which can comprehensively reflect the antioxidant capacity $[14,15]$.Cancer causing carcinogens are often accompanied by the increasing of free radical, elevated cancer promoting process has free radical, free radical in the early formation showed a significant increasing trend of tumor. Antioxidants can scavenge oxygen free radicals and lipid free radicals, prevent lipid peroxidation production and blocking lipid peroxidation chain reaction, antioxidants play an active role in the prevention and treatment of cancer, have been reported in the literature of Figs have anti-cancer anticancer effect better, and that the content of aroma constituents and saponins, glycosides are one of the active components in the anticancer. Our study suggests that, the FCPS content is high, and there is a good antioxidant, can significantly increase the SOD, CAT, GSH-Px activity in blood, and decrease the level of LPO in plasma, liver, and brain homogenate.

In recent years, a large number of studies show that in addition to protein, nucleic acid, carbohydrate is outside of a kind of important biological macromolecules. The study found that plant polysaccharides not only has anti-cancer, anti-inflammatory, antiviral, anti-aging and other pharmacological activities, and the eye has a good scavenging effects on oxygen free radicals of various active[16]. Cancer although occurring in various age stages are, but still with the elderly have more. FCPS through its antioxidant effect good not only played the role of anti-aging, but also has good preventive and therapeutic effects on cancer occurrence and development. The polysaccharide component is the main activity of the main antioxidant, anticancer, anti-ageing ingredients.

Fig cultivation is not only very edible value, the Fig tree and leaves a lot of also has medicinal value, French scientists study found, long-term living in Fig planting area near the crowd cancer incidence rates are particularly low. In addition to the fig in planting with its unique fragrant flavor does not attract pests, no need to spray pesticides, is a natural green agricultural products. On the other hand, figs, high yield, can deep processing making tea, wine and other side products, therefore this 
research is based on the fig nutritional value and medicinal value, the fig development has important economic significance and social benefits.

\section{Acknowledgments}

The research work was supported by Henan Province outstanding scientific and technological innovation team (TCJ2014-391); science and technology innovation team of Zhengzhou city (131PCXTD612); the natural science foundation of Henan province (132300410019).

\section{References}

1. Z.G. Wang, D. He, H. Jin, Progress in modern biomedicine, 11, 015 (2010).

2. L.N. Wang, Q. Wang, M.S. Miao, China Journal of Chinese Medicine, 25 (2010).

3. M. EKislev, A. Hartmann, Ofer Bar-Yosef. Early Domesticated Fig in the jordan valley science, 312 (2006):1372.

4. J.Z. Chai, Y.Y. Huang, G.L. Yuan, Chinese Traditional Patent Medicine, 38, 8 (2016).

5. Y.B. Zai, B. Zhao, Modern distance education of Chinese traditional medicine, 13, 6 (2015).
6. Z.L. Liu, Journal of Applied Sciences, 13, 21, (2013).

7. R.N. Guo, M.X. Ni, ChemistryBioengineering, 32, 3 (2015).

8. Q. Chen, People's Medical Publishing House (1994).

9. M.S. Miao, Chinese press of traditional Chinese Medicine (1997) 249.

10. M.S. Miao, W.Y. Xin, M. Bai, Pharmacology and clinical, 6, 7 (2014).

11. Y.Y. Zhang, Y.H. Wang, Y. Shi, Journal of Zhejiang Chinese Medicine University, 38, 3 (2014).

12. M.T. Mohammadi, R. Amini, Z. Jahanbakhsh, Iran Biomed, 17, 3, (2013).

13. S.S. Qiu, C.C. Jiang, Z.Z. Tan, A preliminary study on the fig crude polysaccharide in vitro antioxidant capacity. Lishizhen medicine and Chinese Medicine, 22, 7 (2011).

14. N. Shi, J. Su, Z.B. Yang, Chinese journal of new drugs, 23, 5 (2014).

15. K.G. Zhu, X.D. Song, Y.P. Sun, Journal of Mechanical Engineering Research and Developments, 39, 1 (2016): p. 239-243.

16. S.S. Qiu, T. Zhou, C.C. Jiang, Food and machinery, 27, 1 (2011). 